\title{
The flow of a class of Oldroyd fluids around a re-entrant corner
}

\author{
Marco A. Fontelos \\ Departamento de Matemática Aplicada \\ Universidad Rey Juan Carlos \\ Móstoles, Madrid 28933. \\ Avner Friedman \\ University of Minnesota \\ Department of Mathematics \\ Minneapolis, Minnesota 55410.
}

February 10, 2000

\begin{abstract}
The singularity near a re-entrant corner of Oldroyd-B fluid has been studied in the literature, at least for re-entrant corner $\gamma=270^{\circ}$. It was shown that the flow develops singularities which are self-similar, very much like the Newtonian singularities. This paper considers the case of a general Oldroyd model and a general re-entrant corner. Under some assumptions on the coefficients of the Oldroyd model (which exclude the Oldroyd-B model) it is shown that the singularity is a sum of a Newtonian singularity plus a lower order non-Newtonian singularity; the latter singularity is not a similarity solution, and, in particular, it is highly oscillatory near the downstream wall, in the vicinity of the corner.
\end{abstract}

\section{Introduction}

When fluid flows around a sharp re-entrant corner the stress develops a singularity. For Newtonian 2-D flow the singularity is $O\left(r^{\lambda-1}\right)$, where $\lambda$ depends on the reentrant angle $\gamma$, and $0<\lambda<1$; more precisely, the stream function $\psi$ has the similarity form

$$
-r^{\lambda+1} f(\theta) \quad(f>0)
$$


where $r$ and $\theta$ are the polar coordinates centered at the corner and $f(\theta)$ vanishes quadratically along the wall; $\psi$ may also be a linear combination of such similarity solutions.

For non-Newtonian fluids, the shape of the singularity near the corner is generally unknown. There are however several papers which, for $\gamma=270^{0}$, suggest a similarity solution of the form (1.1). For power law with viscosity $\gamma=|D|^{n-1}$, Henrickson and Hassager (cf. [5]) found that the stress singularity is like $r^{\beta}$ where $\beta=-0.31$ for a thinning fluid with $n=0.5$ and $\beta=-0.54$ for $n=1.5$. For suspensions of rigid rods aligned with the flow, Keiller and Hinch (cf. [8]) found $\beta=-0.42$ for effective concentration of rods $\phi=5 \%$, and $\beta=-0.38$ for $\phi=20 \%$. In the case of Upper-convected Maxwell fluids, Libscomb et al. (cf. [9]) and Coates et al. (cf. [1]) obtained numerical results suggesting a stress singularity between $r^{-0.90}$ and $r^{-1.0}$ at a Deborah number of 0.65 . More general Oldroyd-B fluids were considered by Davies and Devlin (see [2]) who identified, in particular (again for $\gamma=270^{0}$ ), solutions with stress singularity $r^{-0.613}$. On the other hand, Hinch (see [6]) established for Oldroyd-B fluids (and $\gamma=270^{\circ}$ ) a solution of the form (1.1) under some additional assumptions very close to the walls.

In this paper we consider a general family of Oldroyd fluids and any re-entrant angle $\gamma, \pi<\gamma<2 \pi$. We show, under some conditions on the parameters of the Oldroyd model, that near the corner the non-Newtonian stress $\tau$ is given by

$$
\boldsymbol{\tau}=\frac{\alpha_{2}}{\alpha_{1}} \mathbf{D}+\mathbf{T}
$$

where $\mathbf{D}$ denotes the Newtonian strain tensor with asymptotic behavior near the corner

$$
\mathbf{D} \sim A_{1} r^{\lambda_{1}-1}+o\left(r^{\lambda_{1}-1}\right) \quad\left(A_{1} \text { a matrix independent of } r\right),
$$

$\alpha_{1}, \alpha_{2}$ are two relaxation times which depend on the particular fluid under consideration, and $\mathbf{T}$ has a non-Newtonian and non-selfsimilar lower order singularity. $\mathbf{T}$ is not self-similar and has a rather complex structure. Near the upstream wall $\Gamma_{1}$ (see Figure 1.1) $\mathbf{T}$ has the form

$$
\mathbf{T}(r, \theta) \sim A_{2}\left(\frac{r^{1-\lambda_{1}}}{\theta}\right)^{-\frac{2}{3-\lambda_{1}} b} \quad(A \text { constant matrix })
$$

where $b$ is a parameter determined by the coefficients of the Oldroyd model. Similarly, near the downstream wall $\Gamma_{2}$,

$$
\mathbf{T}(r, \theta) \sim C_{1} \mathbf{T}_{c}(r, \theta)+C_{2} \mathbf{T}_{d}(r, \theta) \quad \text { if } \rho \equiv \frac{r^{1-\lambda_{1}}}{(\gamma-\theta)} \text { is bounded }
$$




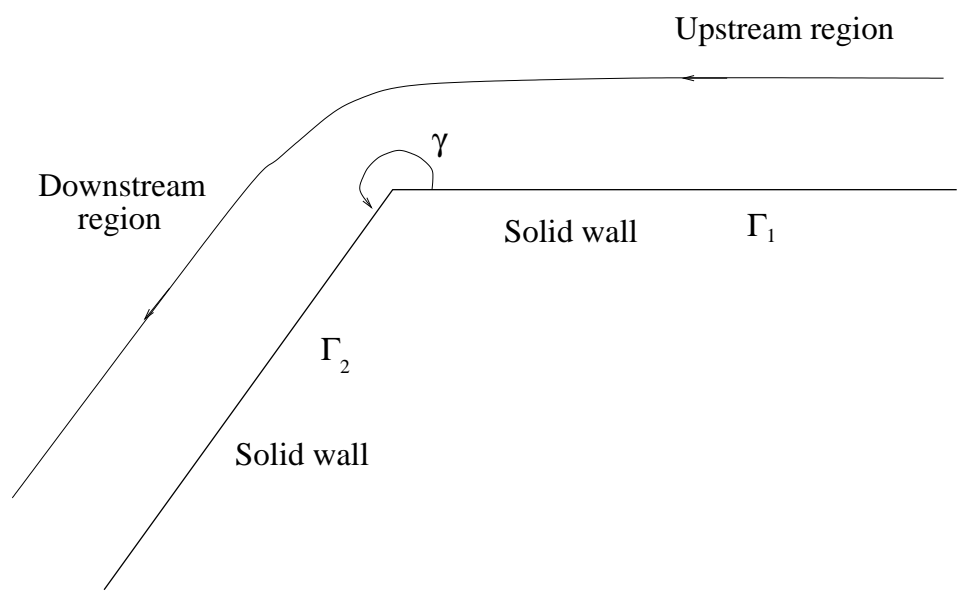

Figure 1.1: The flow around a corner

with

$$
\begin{aligned}
& \mathbf{T}_{c}(r, \theta) \sim A_{0} \rho^{\frac{7-5 \lambda_{1}}{\left(3-\lambda_{1}\right)\left(\lambda_{1}+1\right)}} e^{\frac{2 \nu}{\lambda_{1}+1} \frac{\rho}{r^{1-\lambda_{1}}} i} r^{-3\left(1-\lambda_{1}\right)} \frac{b}{\lambda_{1}+1} \\
& \mathbf{T}_{d}(r, \theta) \sim\left(A_{1} e^{-\frac{2 \nu}{\left(\lambda_{1}+1\right)(\gamma-\theta)} i}+A_{2} e^{\frac{2 \nu}{\left(\lambda_{1}+1\right)(\gamma-\theta)} i}\right) \rho^{\frac{b}{\lambda_{1}}} r^{\frac{\lambda_{1}-1}{\lambda_{1}} b}
\end{aligned}
$$

where $A_{i}(i=0,1,2)$ are constant matrices and $\nu$ is a real parameter determined by the coefficients of the Oldroyd model; $\mathbf{T}(r, \theta)$ is bounded when $\rho \rightarrow \infty$. In the region near the corner and away from $\Gamma_{1}$ and $\Gamma_{2}, \mathbf{T}$ has the same behavior as in (1.2).

\section{The model}

We consider a fluid that satisfies the conservation of momentum and mass equations

$$
\begin{aligned}
(\vec{v} \cdot \nabla) \vec{v} & =-\nabla p+\frac{1}{\operatorname{Re}} \operatorname{div} \boldsymbol{\tau} \text { in } \Sigma \\
\nabla \cdot \vec{v} & =0 \quad \text { in } \Sigma
\end{aligned}
$$


where $\tau$ satisfies the constitutive law of Oldroyd models in the Johnson-Segalman form:

$$
\boldsymbol{\tau}+\alpha_{1}\left(\boldsymbol{\tau}_{(1)}+c(\operatorname{Tr} \boldsymbol{\tau}) \mathbf{D}+d(\boldsymbol{\tau}: \mathbf{D}) \mathbf{I}\right)=\mathbf{D}+\alpha_{2}\left(\mathbf{D}_{(1)}+d(\mathbf{D}: \mathbf{D}) \mathbf{I}\right),
$$

and

$$
\begin{gathered}
D_{i j}=\frac{1}{2}\left(\frac{\partial v_{i}}{\partial x_{j}}+\frac{\partial v_{j}}{\partial x_{j}}\right) \\
\boldsymbol{\tau}: \mathbf{D}=\sum_{i, j} \tau_{i j} D_{i j}, \mathbf{D}: \mathbf{D}=\sum_{i, j} D_{i j} D_{i j},
\end{gathered}
$$

$c$ and $d$ are real parameters, $\alpha_{1}$ and $\alpha_{2}$ are positive constants ("relaxation times"),

$$
\begin{aligned}
\boldsymbol{\tau}_{(1)} & =(\vec{v} \cdot \nabla) \boldsymbol{\tau}-\mathbf{W} \boldsymbol{\tau}+\boldsymbol{\tau} \mathbf{W}-a(\mathbf{D} \boldsymbol{\tau}+\boldsymbol{\tau} \mathbf{D}), \\
\mathbf{D}_{(1)} & =(\vec{v} \cdot \nabla) \mathbf{D}-\mathbf{W D}+\mathbf{D W}-2 a \mathbf{D D},
\end{aligned}
$$

are the tensorial material derivatives of $\tau$ and $D$, and

$$
\mathbf{W}=\frac{1}{2}\left(\nabla \vec{v}-(\nabla \vec{v})^{T}\right)
$$

where $\vec{v}=\left(v_{x}, v_{y}\right)$; Re is the Reynolds number.

In the sequel we shall assume that

$$
1+d a-d c-a^{2}+a c>0
$$

This assumption implies that, in (3.9), $\nu$ is a real number, which will be used in showing that the leading order singularity is Newtonian. The class of fluids studied here includes the Jeffreys model $(a=c=d=0)$ but not the Oldroyd A and B models $(c=d=0,|a|=1)$.

Following other authors (cf. [10], for instance), we first replace in the definitions of $\boldsymbol{\tau}_{(1)}$ and $\mathbf{D}_{(1)}$, the velocity $\vec{v}$ by a Newtonian velocity $\overrightarrow{v_{0}}$. This will simplify the calculations, and will also make the analysis for the case (2.4), later on, more transparent. Thus we shall first work with the non-Newtonian law

$$
\boldsymbol{\tau}+\alpha_{1}\left(\boldsymbol{\tau}(1)+c(\operatorname{Tr} \boldsymbol{\tau}) \mathbf{D}_{0}+d\left(\boldsymbol{\tau}: \mathbf{D}_{0}\right) \mathbf{I}\right)=\mathbf{D}+\alpha_{2}\left(\mathbf{D}_{(1)}+d\left(\mathbf{D}: \mathbf{D}_{0}\right) \mathbf{I}\right),
$$

where

$$
\begin{aligned}
\boldsymbol{\tau}_{(1)} & =\left(\vec{v}_{0} \cdot \nabla\right) \boldsymbol{\tau}-\mathbf{W}_{0} \boldsymbol{\tau}+\boldsymbol{\tau} \mathbf{W}_{0}-a\left(\mathbf{D}_{0} \boldsymbol{\tau}+\boldsymbol{\tau} \mathbf{D}_{0}\right) \\
\mathbf{D}_{(1)} & =\left(\vec{v}_{0} \cdot \nabla\right) \boldsymbol{\tau}-\mathbf{W}_{0} \mathbf{D}+\mathbf{D} \mathbf{W}_{0}-a\left(\mathbf{D}_{0} \mathbf{D}+\mathbf{D D}_{0}\right)
\end{aligned}
$$


and

$$
\begin{aligned}
\mathbf{W}_{0} & =\frac{1}{2}\left(\nabla \vec{v}_{0}-\left(\nabla \vec{v}_{0}\right)^{T}\right), \\
\mathbf{D}_{0} & =\frac{1}{2}\left(\nabla \vec{v}_{0}+\left(\nabla \vec{v}_{0}\right)^{T}\right), \\
\nabla \vec{v}_{0} & =\left(\begin{array}{ll}
\frac{\partial v_{0, x}}{\partial x} & \frac{\partial v_{0, x}}{\partial y} \\
\frac{\partial v_{0, y}}{\partial x} & \frac{\partial v_{0, y}}{\partial y}
\end{array}\right) .
\end{aligned}
$$

The Newtonian velocity is given by

$$
v_{0, x}=\frac{\partial \psi_{0}}{\partial y}, v_{0, y}=-\frac{\partial \psi_{0}}{\partial x}
$$

and for simplicity we first take $\psi_{0}$ as in (1.1), i.e.,

$$
\psi_{0}=-r^{1+\lambda_{1}} f(\theta), f(\theta)>0
$$

where $f(\theta)$ vanishes quadratically at both $\theta=0$ and $\theta=\gamma$. Here the exponent $\lambda_{1}$ is the smallest solution of

$$
\lambda_{1} \sin \gamma+\sin \left(\lambda_{1} \gamma\right)=0
$$

such that the square of the strain tensor associated to $\vec{v}_{0}$ is integrable in a neighborhood of the corner, i.e., $0<\lambda_{1}<1$. It is easy to see that there is precisely one value of $\lambda_{1}$ for each $\gamma$. Indeed, consider the function

$$
g(\lambda)=\lambda \sin \gamma+\sin (\lambda \gamma)
$$

It is clear that $g\left(\frac{\pi}{\gamma}\right)<0, g(1)<0$ and, since $g^{\prime \prime}(\theta)=-\lambda^{2} \sin (\lambda \gamma)>0$ if $\frac{\pi}{\gamma}<\lambda<1, g(\lambda)$ cannot take a maximum in this interval and, consequently, $g(\lambda)<0$ if $\frac{\pi}{\gamma} \leq \lambda \leq 1$. On the other hand $g^{\prime \prime}(\lambda)<0$ if $0<\lambda<\frac{\pi}{\gamma}$ and $g^{\prime}(0)>0$, so that $g^{\prime}(\lambda)>0$ if $0<\lambda<\lambda_{0}, g^{\prime}(\lambda)<0$ if $\lambda_{0}<\lambda<\frac{\pi}{\gamma}$ for some $0<\lambda_{0}<\frac{\pi}{\gamma}$ (if $g^{\prime}(\lambda)>0$ in the entire interval $0<\lambda<\frac{\pi}{\gamma}$ then $g\left(\frac{\pi}{\gamma}\right)>0$, a contradiction). It follows that $g(\lambda)$ has a unique zero in the interval $0<\lambda<\frac{\pi}{\gamma}$.

Later on (in Section 5) we shall discuss the general case of the Oldroyd model where $(2.6),(2.8)$ are replaced by $(2.3),(2.4)$. 


\section{The upstream stress}

In view of (2.2) we can introduce the stream function $\psi$ by

$$
v_{x}=\psi_{y} \quad, \quad v_{y}=-\psi_{x} .
$$

Setting

$$
\mathbf{T}=\boldsymbol{\tau}-\frac{\alpha_{2}}{\alpha_{1}} \mathbf{D}
$$

or, equivalently,

$$
T \equiv\left(\begin{array}{c}
T_{x x} \\
T_{y y} \\
T_{x y}
\end{array}\right)=\left(\begin{array}{c}
\tau_{x x} \\
\tau_{y y} \\
\tau_{x y}
\end{array}\right)-\frac{\alpha_{2}}{\alpha_{1}}\left(\begin{array}{c}
D_{x x} \\
D_{y y} \\
D_{x y}
\end{array}\right) \equiv \tau-\frac{\alpha_{2}}{\alpha_{1}} D
$$

and taking the curl in the Navier-Stokes equation, we get the following equation for $\psi$ :

$$
\Delta^{2} \psi=\frac{\alpha_{1}}{\alpha_{2}} \operatorname{Re} \operatorname{curl}(\vec{v} \cdot \nabla) \vec{v}-\frac{\alpha_{1}}{\alpha_{2}} \operatorname{curl} \operatorname{div}(\mathbf{T}) .
$$

In order to rewrite the constitutive law in a more convenient way, we introduce the matrix

$$
\begin{aligned}
& A=A(\vec{v})= \\
& \left(\begin{array}{ccc}
(-2 a+c+2 d) \frac{\partial v_{x}}{\partial x} & (c-2 d) \frac{\partial v_{x}}{\partial x} & \frac{\partial v_{y}}{\partial x}-\frac{\partial v_{x}}{\partial y}+(d-a)\left(\frac{\partial v_{y}}{\partial x}+\frac{\partial v_{x}}{\partial y}\right) \\
(-c+2 d) \frac{\partial v_{x}}{\partial x} & (2 a-c-2 d) \frac{\partial v_{x}}{\partial x} & -\frac{\partial v_{y}}{\partial x}+\frac{\partial v_{x}}{\partial y}+(d-a)\left(\frac{\partial v_{y}}{\partial x}+\frac{\partial v_{x}}{\partial y}\right) \\
-\frac{1+a-c}{2} \frac{\partial v_{y}}{\partial x}+\frac{1-a+c}{2} \frac{\partial v_{x}}{\partial y} & \frac{1-a+c}{2} \frac{\partial v_{y}}{\partial x}-\frac{1+a-c}{2} \frac{\partial v_{x}}{\partial y} & 0
\end{array}\right)
\end{aligned}
$$

Then, as in [4], the constitutive law (2.6) takes the form

$$
T+\alpha_{1}\left(\left(\vec{v}_{0} \cdot \nabla\right) T+A\left(\vec{v}_{0}\right) T\right)=\left(1-\frac{\alpha_{1}}{\alpha_{2}}\right) D .
$$

We try to solve the hyperbolic system (3.3) by integrating along the streamlines of $\overrightarrow{v_{0}}$, i.e., along the curves $(x(s), y(s))$ with

$$
\frac{d x}{d s}=\frac{\partial \psi_{0}}{\partial y}, \frac{d y}{d s}=-\frac{\partial \psi_{0}}{\partial x}
$$

As in [4] it is convenient to decompose $T$ in the form

$$
T=U V
$$


where $U$ is a $3 \times 3$ matrix satisfying

$$
\frac{d}{d s} U+A\left(\vec{v}_{0}\right) U=0
$$

and $V$ is a vector satisfying

$$
V+\alpha_{1} \frac{d V}{d s}=\left(1-\frac{\alpha_{1}}{\alpha_{2}}\right) U^{-1} D_{0}
$$

We shall analyze $U$ and $V$ near the corner, first near the upstream wall (this section) and then near the downstream wall (in the next section).

It will be convenient to identify the upstream wall with $\theta=0$, the flow region with $0<\theta<\gamma$, and the downstream wall with $\theta=\gamma$. Thus, for $\theta$ near 0 ,

$$
\frac{d}{d s}=\frac{d \theta}{d s} \frac{d}{d \theta}=-\frac{1}{r} \psi_{r} \frac{d}{d \theta} \approx\left(\lambda_{1}+1\right) r^{\lambda_{1}-1} \theta^{2} \frac{d}{d \theta}
$$

and

$$
\begin{gathered}
A\left(\vec{v}_{0}\right) \approx r^{\lambda_{1}-1} \times \\
\times\left[\left(\begin{array}{ccc}
2 \lambda_{1}(2 a-c-2 d) \theta & 2 \lambda_{1}(-c+2 d) \theta & 2+2(d-a) \\
2 \lambda_{1}(c-2 d) \theta & 2 \lambda_{1}(-2 a+c+2 d) \theta & -2+2(d-a) \\
-(1-a+c) & -(-1-a+c) & 0
\end{array}\right)+O\left(\theta^{2}\right)\right] \\
\equiv r^{\lambda_{1}-1}\left[G(\theta)+O\left(\theta^{2}\right)\right] .
\end{gathered}
$$

Therefore the equation for $U$ can be written as

$$
\left(\lambda_{1}+1\right) \frac{d U}{d\left(\theta^{-1}\right)}+G(\theta) U=0
$$

In order to integrate this matrix equation, we first find the eigenvalues of $G$ :

$$
\begin{gathered}
\lambda_{0}=-2 b \theta+o\left(\theta^{2}\right) \\
\lambda_{ \pm}= \pm 2 i \sqrt{\left(1+d a-d c-a^{2}+a c\right)}+b \theta+o\left(\theta^{2}\right) \equiv \pm 2 \nu i+b \theta+o\left(\theta^{2}\right)
\end{gathered}
$$

where

$$
b=2 \lambda_{1} d \frac{a-c}{1+d a-d c+a c-a^{2}} ;
$$

$b$ can be positive or negative. Note that the assumption (2.5) guarantees that $\nu$ is real. 
We diagonalize $G(\theta)$ by means of a matrix $M_{-}$, that is,

$$
M_{-}(\theta) G(\theta) M_{-}^{-1}(\theta) \quad \text { is diagonal ; }
$$

$M_{-}$and $M_{-}^{-1}$ are both bounded matrices, and a simple computation shows that

$$
M_{-}(0)=\left(\begin{array}{ccc}
1 & -\frac{-1-d+a}{1-d+a} & 0 \\
1 & \frac{1+a-c}{-1+a-c} & \frac{2 \nu i}{-1+a-c} \\
1 & \frac{1+a-c}{-1+a-c} & \frac{-2 \nu i}{-1+a-c}
\end{array}\right) .
$$

We can then solve $U$ and obtain near $\Gamma_{1}$ :

$U(\theta)=M_{-}^{-1}\left(\begin{array}{ccc}\theta^{\frac{2 b}{\lambda_{1}+1}} & 0 & 0 \\ 0 & \theta^{-\frac{b}{\lambda_{1}+1}} e^{\frac{2 \nu}{\lambda_{1}+1} \frac{1}{\theta} i} & 0 \\ 0 & 0 & \theta^{-\frac{b}{\lambda_{1}+1}} e^{-\frac{2 \nu}{\lambda_{1}+1} \frac{1}{\theta} i}\end{array}\right) M_{-} C_{-}+o\left(\theta^{\delta}\right) \quad, \delta>0$

where $C_{-}$is any nonsingular matrix.

We next solve for $V$ from (3.6):

$$
V(s)=e^{\frac{-s+s_{0}}{\alpha_{1}}} V\left(s_{0}\right)+\left(1-\frac{\alpha_{2}}{\alpha_{1}}\right) \alpha_{1}^{-1} \int_{s_{0}}^{s} e^{\frac{-s+\sigma}{\alpha_{1}}} U^{-1}(\sigma) D(x(\sigma), y(\sigma)) d \sigma .
$$

and therefore

$T=U V=e^{\frac{-s+s_{0}}{\alpha_{1}}} U(s) V\left(s_{0}\right)+\left(1-\frac{\alpha_{2}}{\alpha_{1}}\right) \alpha_{1}^{-1} \int_{s_{0}}^{s} e^{\frac{-s+\sigma}{\alpha_{1}}} U(s) U^{-1}(\sigma) D(x(\sigma), y(\sigma)) d \sigma$

We shall consider only the integral term, since (in (3.12)) the contribution to $V(s)$ from $e^{\frac{-s+s_{0}}{\alpha_{1}}} V\left(s_{0}\right)$ is exponentially small compared with the contribution coming from the integral. In order to estimate the integral in (3.13), we proceed as in [4]. The main term in the integral in (3.12) is obtained by replacing $D$ by $D_{0}$ and, thus, the principal term in $T$, near $\Gamma_{1}$, is given by the constant $\left(1-\frac{\alpha_{2}}{\alpha_{1}}\right) \alpha_{1}^{-1}$ times the integral

$$
I \equiv \int_{s_{0}}^{s} e^{\frac{-s+\sigma}{\alpha_{1}}} U(s) U^{-1}(\sigma) D_{0}(x(\sigma), y(\sigma)) d \sigma .
$$

Along any streamline $(x(s), y(s))$ the parameter $\theta$ determines uniquely the parameter $s$, and we shall locally write $U(\theta)$ instead of $U(s)$. Substituting $s=s(\theta)$ one gets (recall (3.7))

$$
I=C_{1} \int_{\theta_{0}}^{\theta_{f}} e^{-C_{2} \int_{\widetilde{\theta}}^{\theta_{f}} r^{1-\lambda_{1}}\left(\theta^{\prime}\right) \theta^{\prime-2} d \theta^{\prime}} U\left(\theta_{f}\right) U^{-1}(\widetilde{\theta}) D_{0}(r(\widetilde{\theta}), \widetilde{\theta}) r^{1-\lambda_{1}}(\widetilde{\theta}) \widetilde{\theta}^{-2} d \widetilde{\theta}
$$


where $r(\widetilde{\theta})$ is given implicitly by $\psi(r, \theta)=-r_{f}^{1+\lambda_{1}} \theta_{f}^{2}$. Here $\left(r_{f}, \theta_{f}\right)$ is the point where $T$ is evaluated, and $\left(r_{0}, \theta_{0}\right)$ is a reference point (belonging to the same streamline) that can be taken at $r_{0}=\delta_{0}$ (fixed and small) and $\theta_{0}$ such that $r_{f}^{1+\lambda_{1}} \theta_{f}^{2}=r_{0}^{1+\lambda_{1}} \theta_{0}^{2}$, i.e.,

$$
\theta_{0}=\left(\frac{r_{f}}{\delta}\right)^{\frac{1+\lambda_{1}}{2}} \theta_{f}
$$

The term $D_{0}(r(\widetilde{\theta}), \widetilde{\theta}) r^{1-\lambda_{1}}(\widetilde{\theta})$ is $O(1)$ or $O(\theta)$ (depending on the component of $D_{0}$ considered) while $U\left(\theta_{f}\right) U^{-1}(\widetilde{\theta})$ can be either

$$
\left(\frac{\widetilde{\theta}}{\theta_{f}}\right)^{-2 \frac{b}{\lambda_{1}+1}} \quad \text { or }\left(\frac{\widetilde{\theta}}{\theta_{f}}\right)^{\frac{b}{\lambda_{1}+1}} e^{ \pm \frac{2 \nu}{\lambda_{1}+1}\left(\frac{1}{\tilde{\theta}}-\frac{1}{\theta_{f}}\right) i} .
$$

Note that,

$$
\begin{gathered}
\int_{\widetilde{\theta}}^{\theta_{f}} r^{1-\lambda_{1}}\left(\theta^{\prime}\right) \theta^{\prime-2} d \theta^{\prime} \sim r_{f}^{1-\lambda_{1}} \theta_{f}^{2 \frac{1-\lambda_{1}}{1+\lambda_{1}}} \int_{\widetilde{\theta}}^{\theta_{f}} \theta^{\prime-2-2 \frac{1-\lambda_{1}}{1+\lambda_{1}}} d \theta^{\prime} \\
\sim C r_{f}^{1-\lambda_{1}} \theta_{f}^{2 \frac{1-\lambda_{1}}{1+\lambda_{1}}}\left(\widetilde{\theta}^{\frac{-3+\lambda_{1}}{1+\lambda_{1}}}-\theta_{f}^{\frac{-3+\lambda_{1}}{1+\lambda_{1}}}\right) .
\end{gathered}
$$

Hence, if we set $u=\frac{\theta_{f}}{\theta}$, then $I$ becomes a linear combination of three types of integrals

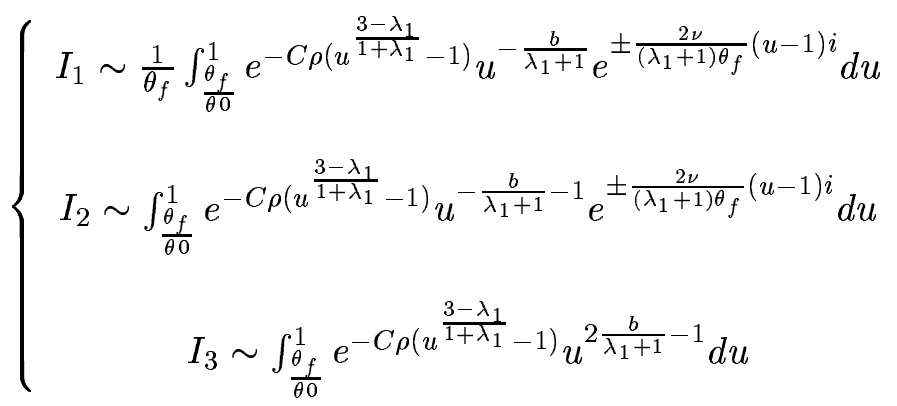

where

$$
\rho=\frac{r_{f}^{1-\lambda_{1}}}{\theta_{f}}
$$

When $\frac{\theta_{f}}{\theta_{0}} \rightarrow \infty$ and $\rho \rightarrow 0$ (which corresponds to a region near the corner and to the highest order singularity of the integrals), the last integral in (3.14) dominates the other two. It follows (by evaluating this integral in (3.14)) that

$$
T\left(r_{f}, \theta_{f}\right) \sim A \rho^{-\frac{2}{3-\lambda_{1}} b}
$$


where $A$ is a constant vector. If we fix $\theta_{f}=\theta_{1}>0$, then along the line $\theta=\theta_{1} T$ has an asymptotic behavior of the form

$$
T\left(r_{f}, \theta_{1}\right) \sim C r^{-\frac{2\left(1-\lambda_{1}\right)}{3-\lambda_{1}} b} \quad \text { as } r \rightarrow 0
$$

Note that if $b$ is small enough, namely, if

$$
\frac{2}{3-\lambda_{1}} b<1
$$

then the singularity of $T$ is of lower order than the singularity of the Newtonian strain.

\section{The downstream stress}

Let $0<\theta_{1}<\theta_{2}<\gamma$ where $\theta_{1}<<1, \gamma-\theta_{2}<<1$. In Section 4 we evaluated the stress $T$ in the region $0<\theta<\theta_{1}$. In the region $\theta_{1}<\theta<\theta_{2}$ the matrix $G$ is bounded and, consequently, the matrix $T$ will also be of the form (3.16).

Consider finally the region near the downstream wall were $\theta_{2}<\theta<\gamma$. Analogously to (3.11) we introduce the variables $\vartheta=\gamma-\theta<<1$ and find that

$$
\begin{gathered}
U(\theta)= \\
M_{+}^{-1}\left(\begin{array}{ccc}
\vartheta^{\frac{2 b}{\lambda_{1}+1}} & 0 & 0 \\
0 & \vartheta^{-\frac{b}{\lambda_{1}+1}} e^{\frac{2 \nu}{\lambda_{1}+1} \frac{1}{\vartheta} i} & 0 \\
0 & 0 & \vartheta^{-\frac{b}{\lambda_{1}+1}} e^{-\frac{2 \nu}{\lambda_{1}+1} \frac{1}{\vartheta} i}
\end{array}\right) M_{+} C_{+}+o\left(\vartheta^{\delta}\right) \quad, \delta>0
\end{gathered}
$$

where $M_{+}, M_{+}^{-1}$ are bounded matrices that diagonalize $G(\theta)$ and $C_{+}$is any nonsingular matrix.

The analysis of formula (3.13) in the downstream region yields two contributions: The first due to the convection of the upstream flow (first term at the right hand side of (3.13)) that we will denote as $T_{c}$, and the second due to the integration of the strain (second term at the right hand side of (3.13)) that we will denote as $T_{d}$. The convective contribution along streamlines is given by

$$
T_{c}(s) \sim e^{\frac{-s+s_{0}}{\alpha_{1}}} U(s) U^{-1}\left(s_{0}\right) T\left(s_{0}\right) .
$$

We can parametrize with $\vartheta$, analogously to (3.7). If $s$ corresponds to a pair $\left(r_{f}, \theta_{f}\right)$ and $s_{0}$ to $\left(r, \theta_{2}\right)$, then

$$
d s=C r(\vartheta)^{1-\lambda_{1}} \vartheta^{-2} d \vartheta
$$


and

$$
s-s_{0}=\int_{s_{0}}^{s} d s=\int_{\gamma-\theta_{2}}^{\vartheta_{f}} C r^{1-\lambda_{1}}(\vartheta) \vartheta^{-2} d \vartheta
$$

with

$$
r(\vartheta)=C r_{f} \vartheta_{f}^{\frac{2}{1+\lambda_{1}}} \vartheta^{-\frac{2}{1+\lambda_{1}}}
$$

since along the streamlines $r^{1+\lambda_{1}} \vartheta^{2}=$ Const. $=r_{f}^{1+\lambda_{1}} \vartheta_{f}^{2}$.

Hence

$$
\begin{gathered}
s-s_{0}=C r_{f}^{1-\lambda_{1}} \vartheta_{f}^{-\frac{2\left(1-\lambda_{1}\right)}{1+\lambda_{1}}} \int_{\gamma-\theta_{2}}^{\vartheta_{f}} \vartheta^{2 \frac{1-\lambda_{1}}{1+\lambda_{1}}-2} d \vartheta \\
=C r_{f}^{1-\lambda_{1}} \vartheta_{f}^{-\frac{2\left(1-\lambda_{1}\right)}{1+\lambda_{1}}}\left[\vartheta_{f}^{\frac{1-3 \lambda_{1}}{1+\lambda_{1}}}-\left(\gamma-\theta_{2}\right)^{\frac{1-3 \lambda_{1}}{1+\lambda_{1}}}\right] .
\end{gathered}
$$

We then get

$$
T_{c}\left(r_{f}, \vartheta_{f}\right) \sim C e^{-C r_{f}^{1-\lambda} \vartheta_{f}^{-1}} e^{\frac{2 \nu}{\lambda_{1}+1} \frac{1}{\vartheta_{f}} i} \vartheta_{f}^{-\frac{b}{\lambda_{1}+1}} T\left(r, \theta_{2}\right)
$$

where we have used the estimates

$$
U(s) U^{-1}\left(s_{0}\right) \sim C \vartheta_{f}^{-\frac{b}{\lambda_{1}+1}} e^{\frac{2 \nu}{\lambda_{1}+1} \frac{1}{\vartheta_{f}} i}, e^{\frac{-s+s_{0}}{\alpha_{1}}} \sim C e^{-C r_{f}^{1-\lambda_{1}} \vartheta_{f}^{-1}} .
$$

Note that $T\left(r, \theta_{2}\right)$ is the value of $T(s)$ at $s=s_{0}$ and, since,

$$
r=r_{f} \vartheta_{f}^{\frac{2}{1+\lambda_{1}}}\left(\gamma-\theta_{2}\right)^{-\frac{2}{1+\lambda_{1}}} \quad \text { and } \theta_{2} \text { is fixed, }
$$

we have, as in the proof of (3.16),

$$
T\left(s_{0}\right) \sim C r^{-\frac{2\left(1-\lambda_{1}\right)}{3-\lambda_{1}} b} \sim C r_{f}^{-\frac{2\left(1-\lambda_{1}\right)}{3-\lambda_{1}} b} \vartheta_{f}^{-\frac{4\left(1-\lambda_{1}\right)}{\left(3-\lambda_{1}\right)\left(1+\lambda_{1}\right)} b} .
$$

Hence

$$
T_{c}\left(r_{f}, \vartheta_{f}\right) \sim C e^{-C r_{f}^{1-\lambda_{1}} \vartheta_{f}^{-1}} e^{\frac{2 \nu}{\lambda_{1}+1} \frac{1}{\vartheta_{f}} i} \vartheta_{f}^{-b \frac{7-5 \lambda_{1}}{\left(3-\lambda_{1}\right)\left(\lambda_{1}+1\right)}} r_{f}^{-\frac{2\left(1-\lambda_{1}\right)}{3-\lambda_{1}} b} .
$$

Introducing

$$
\rho=\frac{r_{f}^{1-\lambda_{1}}}{\vartheta_{f}}
$$


we can thus write

$$
\begin{aligned}
T_{c}\left(r_{f}, \vartheta_{f}\right) & \sim C e^{-C \rho} \rho^{\frac{7-5 \lambda_{1}}{\left(3-\lambda_{1}\right)\left(\lambda_{1}+1\right)} b} e^{\frac{2 \nu}{\lambda_{1}+1} \frac{\rho}{r_{f}^{1-\lambda_{1}} i} r_{f}^{-\frac{\left(7-5 \lambda_{1}\right)\left(1-\lambda_{1}\right)}{\left(3-\lambda_{1}\right)\left(\lambda_{1}+1\right)} b} r_{f}^{-\frac{2\left(1-\lambda_{1}\right)}{3-\lambda_{1}} b}} \\
& =C e^{-C \rho} \rho^{\frac{7-5 \lambda_{1}}{\left(3-\lambda_{1}\right)\left(\lambda_{1}+1\right)} b} e^{\frac{2 \nu}{\lambda_{1}+1} \frac{\rho}{r_{f}^{1-\lambda_{1}}} i} r_{f}^{-3\left(1-\lambda_{1}\right) \frac{b}{\lambda_{1}+1}}
\end{aligned}
$$

This singularity is weaker than the Newtonian one if

$$
\frac{3 b}{\lambda_{1}+1}<1
$$

Note that this condition is more restrictive than (3.17).

The integration of the strain results, to the highest order, in a term of the form

$$
\begin{gathered}
I= \\
C_{1} \int_{\theta_{2}}^{\theta_{f}} e^{-C_{2} \int_{\widetilde{\theta}}^{\theta_{f}} r^{1-\lambda_{1}\left(\theta^{\prime}\right)\left(\gamma-\theta^{\prime}\right)^{-2} d \theta^{\prime}} U\left(\theta_{f}\right) U^{-1}(\widetilde{\theta}) D_{0}(r(\widetilde{\theta}), \widetilde{\theta}) r^{1-\lambda_{1}}(\widetilde{\theta})(\gamma-\widetilde{\theta})^{-2} d \widetilde{\theta}}
\end{gathered}
$$

which can be analyzed in the same way as the integral in the upstream region to yield an estimate of the form

$$
|I| \leq C r_{f}^{-\frac{1-\lambda_{1}}{\lambda_{1}} b}
$$

Indeed, if we expand the expression (4.4), identify the different integrals that appear (just as we did in the upstream region) and perform the change of variables $u=\frac{\gamma-\theta_{f}}{\gamma-\theta}$, we obtain linear combinations of integrals of the form

$$
\left\{\begin{array}{c}
I_{1} \sim \frac{1}{\vartheta_{f}} \int_{\frac{\vartheta_{f}}{\gamma-\theta_{2}}}^{1} e^{C \rho\left(u^{\frac{3-\lambda_{1}}{1+\lambda_{1}}}-1\right)} u^{-\frac{b}{\lambda_{1}+1}} e^{ \pm \frac{2 \nu}{\left(\lambda_{1}+1\right) \vartheta_{f}}(u-1) i} d u \\
I_{2} \sim \int_{\frac{\vartheta_{f}}{\gamma-\theta_{2}}}^{1} e^{C \rho\left(u^{\frac{3-\lambda_{1}}{1+\lambda_{1}}}-1\right)} u^{-\frac{b}{\lambda_{1}+1}-1} e^{ \pm \frac{2 \nu}{\left(\lambda_{1}+1\right) \vartheta_{f}}(u-1) i} d u \\
I_{3} \sim \int_{\frac{\vartheta_{f}}{\gamma-\theta_{2}}}^{1} e^{C \rho\left(u^{\frac{3-\lambda_{1}}{1+\lambda_{1}}}-1\right)} u^{2 \frac{b}{\lambda_{1}+1}-1} d u
\end{array}\right.
$$

where $\rho$ is as in (4.1). 
All these integrals are bounded as $\rho \rightarrow \infty$, while for $\rho$ bounded and, in particular, going to zero, $I_{1}$ and $I_{3}$ are bounded, but

$$
\begin{gathered}
I_{2} \sim C_{1} \int_{\frac{\vartheta_{f}}{\gamma-\theta_{2}}}^{1} u^{-\frac{b}{\lambda_{1}}-1} e^{ \pm \frac{2 \nu}{\left(\lambda_{1}+1\right) \vartheta_{f}}(u-1) i} d u \sim C_{2} e^{\mp \frac{2 \nu}{\left(\lambda_{1}+1\right) \vartheta_{f}} i} \vartheta_{f}^{-\frac{b}{\lambda_{1}}} \\
\sim C_{2} e^{\mp \frac{2 \nu}{\left(\lambda_{1}+1\right) \vartheta_{f}} i} \rho^{\frac{b}{\lambda_{1}}} r_{f}^{\frac{\lambda_{1}-1}{\lambda_{1}} b}
\end{gathered}
$$

which is a singular, highly oscillatory term. We conclude that

$$
T_{d}\left(r_{f}, \vartheta_{f}\right) \sim\left(A_{1} e^{-\frac{2 \nu}{\left(\lambda_{1}+1\right) \vartheta_{f}} i}+A_{2} e^{\frac{2 \nu}{\left(\lambda_{1}+1\right) \vartheta_{f}} i}\right) \rho^{\frac{b}{\lambda_{1}}} r_{f}^{\frac{\lambda_{1}-1}{\lambda_{1}} b} .
$$

This singularity is weaker than the Newtonian one if

$$
\frac{b}{\lambda_{1}}<1
$$

Combining this with (4.2) we conclude that to the highest order

$$
T\left(r_{f}, \vartheta_{f}\right) \equiv C_{1} T_{c}\left(r_{f}, \vartheta_{f}\right)+C_{2} T_{d}\left(r_{f}, \vartheta_{f}\right) .
$$

Notice that (4.7) and (4.3) do not imply each other. Also, $\left|T_{c}\right|$ and $\left|T_{d}\right|$ do not majorize each other. In fact, they become of the same order along the curve

$$
r_{f}=\Psi\left(\vartheta_{f}\right) \equiv \vartheta_{f}^{\frac{-5 \lambda_{1}+4 \lambda_{1}^{2}+3}{\lambda_{1}\left(\lambda_{1}+1\right)\left(1-\lambda_{1}\right)}}
$$

with $\left|T_{c}\right|<\left|T_{d}\right|$ for $r_{f}>\Psi\left(\vartheta_{f}\right)$ and $\left|T_{c}\right|>\left|T_{d}\right|$ for $r_{f}<\Psi\left(\vartheta_{f}\right)$; this situation is illustrated in Figure 4.1. We finally note that if $\rho$ is large, i.e.,

$$
\vartheta_{f} \ll r_{f}^{1-\lambda_{1}},
$$

then the right hand side of (4.8) is bounded.

\section{The complete problem}

We shall henceforth assume that the parameter $b$ satisfies the condition (4.7). Then, (3.17) is also satisfied, which implies that the singularity of the nonNewtonian stress $T$ is of smaller order than the Newtonian singularity in the entire vicinity of the corner. We now go back to the equation (3.2) and find the correction $\widetilde{\psi}$ to the stream function $\psi_{0}$. If we write $\Delta^{2}$ and curldiv $(\mathbf{T})$ in polar 


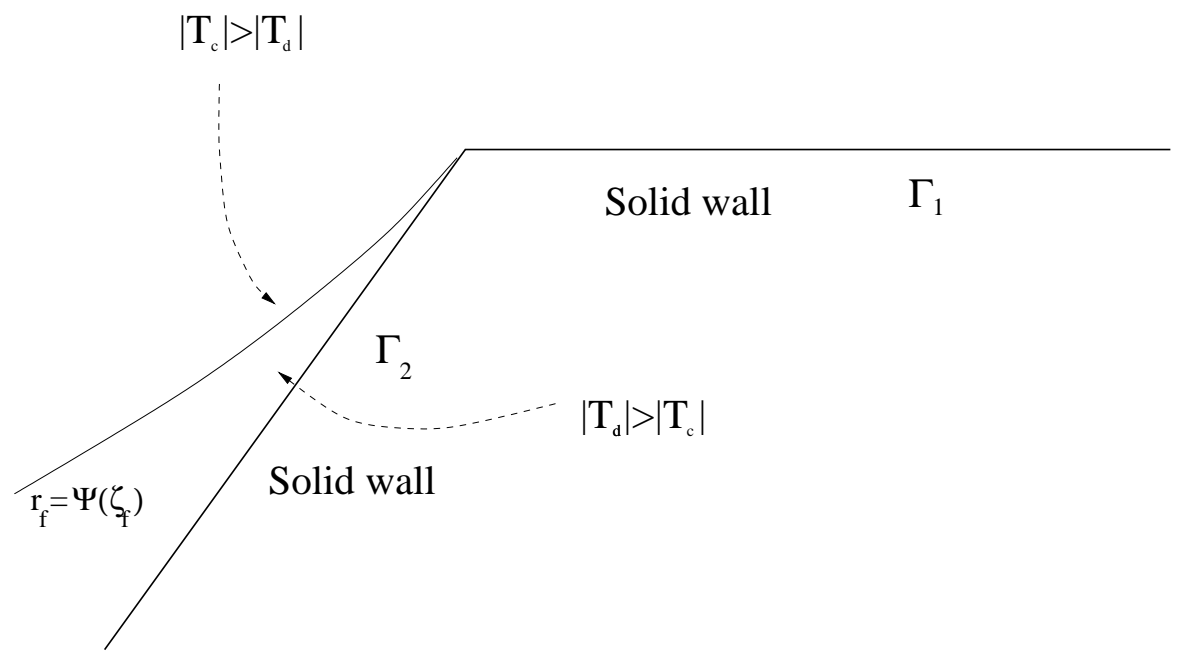

Figure 4.1: The various regions in the downstream area

coordinates and retain only the highest order terms near the corner, we get the equation

$$
\frac{1}{r^{4}} \frac{\partial^{4} \widetilde{\psi}}{\partial \theta^{4}} \sim-\frac{\alpha_{1}}{\alpha_{2}} \frac{1}{r^{2}} \frac{\partial^{2} T_{x y}}{\partial \theta^{2}}
$$

near the upstream wall $\Gamma_{1}$. A similar equation holds near the downstream wall $\Gamma_{2}$ with $\theta$ replaced by $\gamma-\theta$. This combined with the boundary conditions $\widetilde{\psi}=\widetilde{\psi}_{\theta}=0$ at $\theta=0, \gamma$ (and the asymptotic behavior of $T$ obtained in $(3.16),(4.2),(4.6)$ ), yields the bound

$$
|\widetilde{\psi}| \leq C \theta^{2}(\gamma-\theta)^{2} r^{2-3\left(1-\lambda_{1}\right)} \frac{b}{\lambda_{1}+1} .
$$

This correction is small compared to the Newtonian contribution and, therefore, the entire flow should be dominated by the Newtonian term.

If we replace $(2.6),(2.8)$ by $(2.3),(2.4)$ with

$$
\vec{v}=\overrightarrow{v_{0}}+\left(\widetilde{\psi_{y}},-\widetilde{\psi_{x}}\right)
$$

then we can still repeat all the analysis of Sections 3,4 . Indeed, we can perform the decomposition (3.4) and obtain for $U$ the equation (3.5) with $A\left(\overrightarrow{v_{0}}\right)$ replaced 
by $A(\vec{v})$ and for $V$ an equation as in (3.6). In both equations, $s$ is the coordinate along a streamline corresponding to $\vec{v}$. As the non-Newtonian contribution to $\vec{v}$ is small, the corrections introduced by working with (5.2) will only be of lower order than the ones described above in Sections 3,4.

Thus, to the highest order, the stress tensor near the corner will be

$$
\boldsymbol{\tau}=\frac{\alpha_{2}}{\alpha_{1}} \mathbf{D}+\mathbf{T}
$$

where $\mathbf{D}$ is the strain corresponding to $\vec{v}$ and $\mathbf{T}$ is a tensor with the same asymptotic behavior as in Sections 3,4 .

we can now solve the equation (3.2) using the $\mathbf{T}$ which was obtained by working with (5.2). We obtain a new correction $\widetilde{\widetilde{\psi}}$ to the velocity $(5.2)$ and a new stress $T$ in (5.3). We can iterate the process, determining a sequence of velocities and stresses with an asymptotics near the corner given to a leading order by the terms already computed in Sections 3,4. Using analysis as in [4] we expect the process to converge if $\left|1-\frac{\alpha_{2}}{\alpha_{1}}\right|$ is small enough.

\section{Conclusion}

In this paper we have shown that for a general family of Oldroyd flows near a reentrant corner, the stress tensor has the form (5.3) where $\mathbf{D}$ is the strain tensor and $\mathbf{T}$ is a non-Newtonian contribution whose asymptotics has been described in detail. The main restrictions on the coefficients of the model are (2.5) and the inequalities (4.3), (4.7). The singularity of $\mathbf{T}$ is of lower order than the Newtonian one but this singularity has a complex structure. In particular, it is not self-similar, and it is highly oscillatory near the downstream wall.

The inequality (2.5) excludes the case of Oldroyd A and B models, for which $c=d=0$ and $a= \pm 1$. Although our results do not predict the behavior of the singularity, they give an accurate description of the flow for approximating models which belong to the family of Oldroyd flows.

\section{ACKNOWLEDGMENTS}

The first author is grateful for the support from the Institute of Mathematics and its Applications and the Department of Mathematics at Virginia Tech University and has been partially supported by the Spanish Ministry of Education. The second author is partially supported by National Science Foundation Grant DMS \#9970522. 


\section{References}

[1] P. J. Coates, R. C. Armstrong and R. A: Brown, Calculation of steadystate viscoelastic flow through axisymmetric contractions with the EEME formulation, J. Non-Newtonian Fluid Mech., 42 (1992), 141-188.

[2] A. R. Davies and J. Devlin, On corner flows of Oldroyd-B fluids, J. NonNewtonian Fluid Mech., 50 (1993), 173-191.

[3] M. A. Fontelos and A. Friedman. Stationary Non-Newtonian fluid flows in chanel-like and pipe-like domains, Archive Rat. Mech. Anal., to appear.

[4] M. A. Fontelos and A. Friedman. Analysis of the stick-slip problem for NonNewtonian flows., Comm. in Partial Differential Equations, to appear.

[5] P. Henricksen and O. Hassager, J. Rheol., 33 (1989), 865-879.

[6] E. J. Hinch, The flow of an Oldroyd Fluid around a sharp corner, J. NonNewtonian Fluid Mech., 50 (1993), 161-171.

[7] D. D. Joseph, Fluid Dynamics of Viscoelastic Liquids. Springer-Verlag, New York, 1990

[8] R. A: Keiller and E. J. Hinch, Corner flow of a suspension of rigid rods, J. Non-Newtonian Fluid Mech., 40 (1992), 323-335.

[9] G. G. Lipscomb, R. Keunings and M. M. Denn, Implications of boundary singularities in complex geometries, J. Non-Newtonian Fluid Mech., 24 (1987), $85-96$.

[10] M. Renardy. The stresses of an upper convected Maxwell fluid in a Newtonian velocity field near a re-entrant corner. J. non-Newtonian Fluid Mechanics, 50 (1993), 127-134.

[11] T. R. Salamon, D. E. Bornside, R. C. Armstrong and R. A. Brown. Local similarity solutions for the stress filed in the presence of a slip boundary condition. Phys. Fluids, 9 (1997), 1235-1247.

[12] T. R. Salamon, D. E. Bornside, R. C. Armstrong and R. A. Brown. Local similarity solutions for the stress filed of an Oldroyd-B fluid in the partial slip/slip flow. Phys. Fluids, 9 (1997), 2191-2209. 